\title{
Leusonna, die Schutzgöttin des vicus, auf einem Votivbeilchen aus Lausanne-Vidy 1
}

\author{
Jens Bartels
}

Aus dem beim heutigen Lausanne-Vidy gelegenen römischen vicus Leusonna/Lousonna ist inzwischen eine Reihe von Inschriften bekannt. Dazu gehören eine ganze Zahl von Steininschriften, aber auch diverse Kleininschriften auf unterschiedlichen Materialien. Gerade letztere haben bisher zu wenig Aufmerksamkeit gefunden. Eines dieser Objekte ist ein Votivbeilchen, das im Sommer 1984 bei Ausgrabungen des Institut d'archéologie et d'histoire ancienne der Université de Lausanne auf dem Grundstück Rue de Chavannes 29, wohl im Hof des Bâtiment Ouest, gefunden wurde. ${ }^{2} \mathrm{Ca} .150 \mathrm{~m}$ südlich dieses Gebäudes wurde ein kleines Heiligtum freigelegt, aus dem das Beilchen ursprünglich gestammt haben könnte. ${ }^{3}$

Es handelt sich um Axtblatt und Schaftansatz eines ungewöhnlich großen Votivbeilchens: Die Höhe der «Klinge» misst 4,5 cm, die Breite des Axtblattes 6,5 cm, seine Tiefe 2-6 mm. Es ist heute in der Ausstellung des Musée Romain de LausanneVidy zu sehen (inv. VY 84/2814-01). Die Inschrift ist gepunzt und befindet sich wie bei fast allen Votivbeilchen auf der linken Seite des Axtblattes. Sie beginnt am unteren Ende der „Klinge“, verläuft parallel zu dieser und dann entlang der Oberseite des Blattes bis zum Schaftansatz. Der Rest des Textes folgt dann - quasi als zweite Zeile entlang der Unterkante des Axtblattes. Zwischen den Textzeilen sind in der Mitte des Axtblattes Reste einer grossen hedera zu erkennen. Eine weitere kleine hedera trennt in der Textzeile entlang der Oberkante zwei Worte. Die Buchstabenhöhe beträgt 6$7 \mathrm{~mm}$.

Die Lesung der Inschrift ist nicht ganz einfach. Zunächst springt der gut lesbare Schluss der Inschrift ins Auge: Wie auf einer Weihinschrift zu erwarten, sind dort die Buchstaben $V S L M$ zu erkennen, also die Formel v(otum) s(olvit) l(ibens) m(erito). Die grosse gepunzte hedera in der Mitte des Axtblattes ist meines Erachtens als Worttrenner direkt vor dieser Schlussformel zu verstehen. Der Rest der Inschrift ist deutlich schwerer zu entschlüsseln, doch können die bisherigen Ansätze nicht recht überzeugen. Thierry Luginbühl schlug 2006 Folgendes vor:

\section{Ca[turigi] / Caius Istoleus v(otum) s(olvit) l(ibens) $m$ (erito). ${ }^{4}$}

\footnotetext{
1 Das Votivbeil wurde von mir 2011 im Rahmen der von Anne Kolb geleiteten Erstellung einer Neuauflage des den schweizerischen Teil der Germania superior betreffenden Teils von Band XIII des Corpus Inscriptionum Latinarum aufgenommen. Für die Genehmigung und Unterstützung vor Ort danken wir Laurent Flutsch und Sophie Weber.

Die Lesung und erste Überlegungen $\mathrm{zu}$ dieser Inschrift wurden auf der Jahrestagung der Arbeitsgemeinschaft für die provinzialrömische Forschung in der Schweiz (ARS) 2012 in Yverdon vorgestellt. Für Hilfe und Hinweise danke ich Anne Kolb.

${ }^{2}$ So scheint aus der Kombination der Informationen bei PAUNIER 1987a, 1; 63; 141 Nr. 223 zu schliessen zu sein.

${ }^{3}$ Einen Bezug zwischen dem Votivbeilchen und dem Heiligtum stellte erstmals LUGINBÜHL 1996, 15 16 her. Vgl. auch LUGINBÜHL 2006, 67 und LugINBÜHL 2008, 232 sowie CRAMATTE 2008, 272. Zu dem Heiligtum westlich des vicus vgl. PAUNIER 1987b, 119-122; 124; PAUNIER 1989; LUGINBÜHL 2008, 231232; CRAMATTE 2008, 272 Nr. 32.

${ }^{4}$ LUGINBÜHL 2006, 67; vgl. schon LUGINBÜHL 1996, 16.
}

http://www.fera-journal.eu 
(Mars) Caturix ist eine gute Idee, doch gibt es einige Punkte, die Zweifel wecken: Zunächst ist Caturix in den uns bekannten Inschriften bisher lediglich als sekundäres Theonym und niemals als alleinstehender Göttername belegt. ${ }^{5}$ Weiterhin stände $C A$ am rechten Ende der Oberkannte des Axtblattes. Das kann zum einen an dieser Position kaum der Anfang der Inschrift sein, zum anderen markiert an dieser Stelle eine gepunzte Linie den Abschluss des Axtblattes zum Schaft. Es fehlt also eine Stelle, an der der ergänzte Text gestanden haben könnte.

Tatsächlich hatte Laurent Flutsch 2004 bei seiner Vorlage des Beilchens im Begleitband zur Dauerausstellung des Musée Romain de Lausanne-Vidy eine zurückhaltendere Text-Rekonstruktion präsentiert, die der anzunehmenden Leserichtung folgt:

\section{CAIVS YSPOLEVS CA[...]v(otum) s(olvit) l(ibens) m(erito). ${ }^{6}$}

Auch das befriedigt nicht und so äußerte schon 2009 Regula Frei-Stolba Zweifel an den bisherigen Lesungen. ${ }^{7}$ Neben dem sperrigen $C A$ fällt bei beiden Lesungen die Buchstabenfolge ISTOLEVS bzw. YSPOLEVS auf, die offenbar als Nomen oder Cognomen gedeutet wird, ${ }^{8}$ sich aber mit keinem bekannten Namen in Verbindung bringen lässt. Das schwer verständliche $C A$ öffnet den Weg zu einem besseren Verständnis der vorausgehenden Buchstaben. Ca(turigi) oder Ca[turigi] fällt aus den oben genannten Gründen aus. Und tatsächlich zeigte die Autopsie, dass statt $C A$ vielmehr die Buchstaben $O N$ zu lesen sind. Zudem ist zwischen ISTO oder YPSO und LEVS klar eine gepunzte hedera zu erkennen, die hier also zwei Worte trennen muss. Hinter dem Worttrenner ergibt sich damit neu die Buchstabenfolge LEVSON. Diese sechs Buchstaben erinnern an den Ortsnamen der Siedlung, aus der das Votivbeilchen stammt. Dieser wird in den Inschriften entweder Leusonna (AE 1939,209 und 1946, 256; adjektivisch Leusonnensis in AE 1939, 207) oder Lousonna (nur adjektivisch als Lousonnensis belegt: CIL XIII 5026) geschrieben.

Ein Ortsname ist in der vorliegenden Inschriftengattung an dieser Stelle allerdings kaum zu erwarten. Was wir jedoch aus Weihinschriften aus dem keltischen Raum des Imperium Romanum kennen, sind Weihungen an Gottheiten, deren Namen mit denjenigen der Orte, an denen sie gefunden wurden, eng verwandt sind. Am bekanntesten ist vielleicht Nemausus, dem in Nemausus (heute Nîmes) in der Gallia Narbonnensis, eine Reihe von Weihinschriften gewidmet wurden. ${ }^{9}$ Einen ähnlichen Fall stellt darüber hinaus Iuvavus in Iuvavum (heute Salzburg) in Noricum dar. ${ }^{10}$ Wohl ebenfalls in diese Kategorie gehört Vasio in Vasio Vocontiorum (heute Vaison-laRomaine) in der Gallia Narbonnensis, der allerdings auch einmal als Mars Vasio bezeichnet wird. ${ }^{11}$ Treten diese drei Ortsgottheiten in maskuliner Form auf, so lassen sich ebenso solche in femininer Form finden. In Noricum ist Teurnia zu nennen. ${ }^{12}$ Vor

\footnotetext{
${ }^{5}$ Zur Unterscheidung zwischen primären und sekundären Theonymen vgl. u.a. DE BERNARDO STEMPEL 2013.

${ }^{6}$ FLUTSCH 2004, 157 mit der Übersetzung, „À Ca(...?) de la part de Caius Hyspoleus, qui s'est volontiers acquitté de son vœu“. Der Kommentar fügt hinzu: „Il s'agit donc d'un ex-voto à une divinité inconnue: peut-être bien Caturix, le dieu helvète de la guerre, aussi vénéré sous le nom mixte de Mars Caturix." Auf Flutschs Lesung basierte offenbar die website, auf die sich KIERNAN 2009, 140 stützt.

${ }^{7}$ FREI-STOLBA 2009, 39, ohne dies genauer auszuführen.

${ }^{8}$ Vgl. die in Anm. 6 zitierte Übersetzung.

${ }^{9}$ CIL XII 3070; 3072; 3077; 3093-3102; 3132.

${ }^{10}$ AE 2009, 988. Vgl. dazu HaInZMANn 2010 und HAInZMAnN/DE BERnARdo STEMPEL 2012.

${ }^{11}$ ILGN 202 (Vasio); AE 1992, 1202 (stark ergänzt); AE 2003, 1085 (Mars Vasio).

${ }^{12}$ AE 1920, $58=$ ILLPRON 479.
} 
allem aber kennen wir zwei solche femininen Ortsgottheiten aus der unmittelbaren Umgebung von Lausanne: Zum einen Aventia im Hauptort der Gemeinde der Helvetier in Aventicum (heute Avenches) und zum anderen Genava in dem gleichnamigen vicus (heute Genf) auf dem Gebiet der civitas der Allobroger bzw. der Kolonie Vienna. ${ }^{13}$ Es spricht also alles dafür, dass wir in LEVSON den Namen der Gottheit in abgekürzter Form erkennen müssen, der das Votivbeilchen geweiht war.

Das Ende der Inschrift lässt sich also nun als Leuson(nae) v(otum) s(olvit) $m$ (erito) herstellen. Die Rekonstruktion des Anfangs gestaltet sich dagegen schwieriger. Hier können nur vorläufige Überlegungen dazu vorgebracht werden, aus denen Sprachwissenschaftler vielleicht mehr gewinnen können.

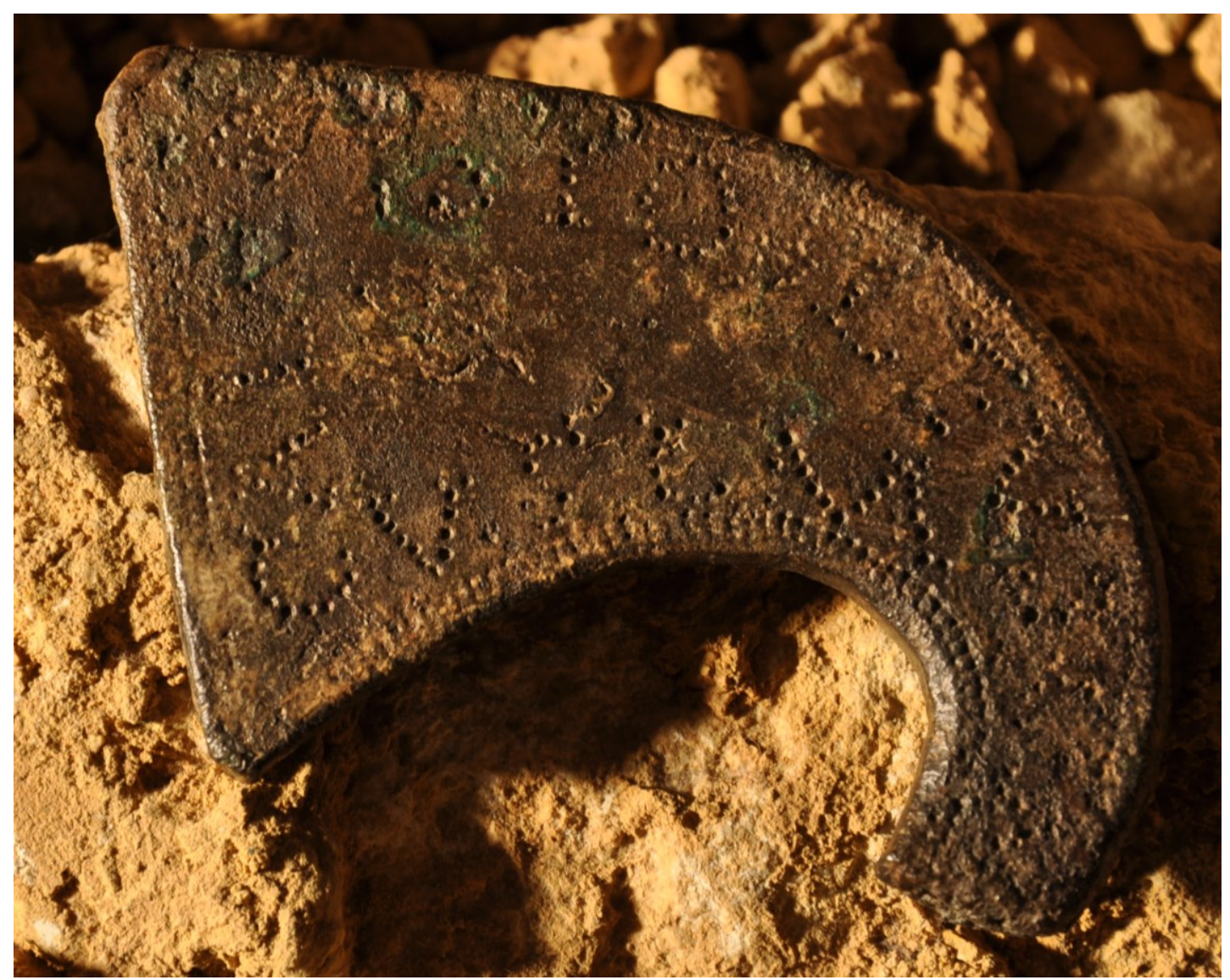

Abb. 1 Votivbeilchen aus Lausanne-Vidy [Foto: Jens Bartels]

Nachdem Weiheformel und die Gottheit am Ende der Inschrift stehen, ist am Anfang am ehesten der Name des Weihenden zu erwarten. ${ }^{14}$ Die ersten drei Buchstaben entlang der „Klinge“ sind klar als $C A L$ zu lesen. Schwer ist hingegen die Deutung des vierten und fünften Buchstabens. Beim vierten scheinen die wenigen erkennbaren Punkte wahrscheinlich auf ein $V \mathrm{zu}$ weisen. Ebenso ist unmittelbar vor der hedera, die Leuson(nae) vom vorherstehenden Wort trennt, relativ sicher $I O \mathrm{zu}$ lesen. Vor $I O$ scheinen die untere Hälfte eines $S$ und darüber einige zu einem $S$ passende Punkte erkennbar zu sein. Von dem Buchstaben davor ist der Ansatz einer senkrechten Haste, aber nicht mehr zu sehen. Bei dem Buchstaben auf der Ecke handelt es sich wohl um ein $A$. Daraus ergibt sich die wahrscheinliche, aber nicht völlig gesicherte Lesung

\footnotetext{
${ }^{13}$ Aventia: CIL XIII 5071-5073; AE 1925,2. Genava: ILN V/3 819-820.

${ }^{14}$ Auf den zwei anderen Votivbeilchen mit etwas längeren Texten stehen die genannten Elemente genau in dieser Reihenfolge: CIL XIII 5172 aus Solothurn und AE 2009, 948 aus Windisch.
}

http://www.fera-journal.eu 
CALVAISSIO. Es würde sich dabei um einen vermutlich keltischen oder keltischstämmigen Individualnamen im Nominativ handeln. ${ }^{15}$ Bei diesem wäre das Schluss-s ausgefallen, was im Spätgallischen häufiger vorkommt. Entsprechend hat man dieses Phänomen bereits bei einer anderen Inschrift aus Lausanne-Vidy erwogen. ${ }^{16}$ Dieser Name ist in dieser Form bisher nicht belegt und das lässt die Lesung unbefriedigend bleiben. Möglichweise gibt es eine Beziehung zu dem im keltischen Raum häufiger belegten cognomen Calvisius, hinter dem ein keltischer Name stecken könnte, der durch Assonanz an den italischen Gentilnamen Calvisius überformt wurde. ${ }^{17}$ Zudem scheint Calvisius auch als Individualname aufzutreten. ${ }^{18}$ Die Sprachwissenschaft wird hier vielleicht weiterkommen.

Die vorläufige neue Lesung des Votivbeilchens aus Lausanne-Vidy lautet also:

Calyạișio(s) (?) · Leuson(nae) v(otum) s(olvit) l(ibens) m(erito).

Calvaisios(?) hat der Leusonna sein Gelübde gerne und nach Verdienst erfüllt.

\footnotetext{
${ }^{15} \mathrm{Zu}$ keltischen Namensbildungen mit dem Element Calv- vgl. HOLDER 1896, 706.

${ }^{16}$ AE 1939,211: Nonio(s) Sul(eis) / suis voto / l(ibens) m(erito) r(estituit?) mit dem Kommentar von COllart/van Berchem 1939, 137. Zu dem Phänomen des Auslassens des End-s in o-stämmigen Nominativen siehe u.a. LAMBERT 1997, 402-406; STIFTER 2010/11; STIFTER 2012, 533-535.

${ }^{17}$ Vgl. z.B. CIL V 4555, 5292, 7028 aus Oberitalien. Zu dem cognomen Calvisius und seinen Belegen vgl. auch KAKOSCHKE 2007, 196. Zum Gentilnamen Calvisius vgl. KAKOSCHKE 2006, 119-120. Vgl. außerdem das je einmal in Oberitalien und Rom belegte nomen gentile Calvasius: CIL V 4748 und VI 31234.

${ }^{18}$ Sicher in RIU VI 1475 aus Aquincum, eventuell auch in CIL III 5519 aus Savaria.
} 


\section{Bibliographie}

Collart/VAn Berchem $1939=$ P. COllart/D. VAn Berchem, Inscriptions de Vidy, Revue historique vaudoise 47, 1939, 127-145

<url: $\quad$ https://www.e-periodica.ch/digbib/view?pid=rhv001\%3A1939\%3A47\%3A\%3A7\#134>.

Cramatte $2008=$ C. Cramatte, Recueil des sanctuaires romains de Suisse, in: D. Castella / M.-F. MeYlan Krause (Hg.), Topographie sacrée et rituels. Le cas d'Aventicum, capitale des Helvètes, Basel 2008, 265-277.

De Bernardo Stempel $2013=$ P. De Bernardo Stempel, Individuality in Celtic Divine Names: Theonyms, Epithets and Theonymic Formulae, in: W. Spickermann (Hg.), Keltische Götternamen als individuelle Option?, Rahden/Westf. 2013, 25-37.

Flutsch 2004 = L. Flutsch, Passé present. Lousonna ou l'Antiquité d'actualité, Gollion 2004.

Frei-Stolba $2009=$ R. Frei-Stolba, Die neue Bauinschrift aus dem Tempelbezirk des Merkurtempels und zwei weitere Weihinschriften aus Vindonissa, Jahresbericht Gesellschaft Pro Vindonissa 2009, 35-41

$<$ url:

https://www.e-periodica.ch/digbib/view?pid=gpv001\%3A2009\%3A0\#41>.

HAINZMANN $2010=$ M. HAINZMANN, Iuvavus - Römischer Stadtgott oder norische Flussgottheit?, Römisches Österreich 33, 2010, 23-33.

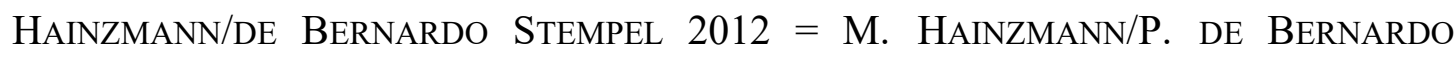
STEMPEL, Iuvavus und Verwandte. Überlieferung, linguistische Kommentierung, Gesamtbeurteilung, Römisches Österreich 34/35, 2011-2012, 51-62.

HOLDER 1896 = A. HOLDER, Alt-celtischer Sprachschatz, Bd. 1, Leipzig 1896.

KAKOSChKe $2006=$ A. KAKOSChKe, Die Personennamen in den zwei germanischen Provinzen. Ein Katalog, Bd. 1: Die Gentilnomina ABILIUS-VOLUSIUS, Rahden/Westf. 2006.

KAKoschKe 2007 = A. KAKOSCHKE, Die Personennamen in den zwei germanischen Provinzen. Ein Katalog, Bd. 2.1: Cognomina ABAIUS-LYSIAS, Rahden/Westf. 2007.

KIERNAN $2009=\mathrm{Ph}$. KIERNAN, Miniature Votive Offerings in the north-west Provinces of the Roman Empire, Mainz/Ruhpolding 2009.

LAMBERT 1997 = P.-Y. LAMBERT, Gaulois tardif et latin vulgaire, Zeitschrift für celtische Philologie 49-50, 1997, 396-413.

LUGINBÜHL $1996=$ Th. LUGINBÜHL, Religion et croyances à Lousonna-Vidy, Mémoire vive 1996, 9-20.

LuginBÜHL 2006 = Th. LugINBÜHL, »Mars Caturix«: numen et sanctuaire du Mars helvète, in: V. BrouQuiER Reddé u.a. (Hg.), Mars en Occident, Rennes 2006, 63-72. 
LUGINBÜHL $2008=$ Th. LUGINBÜHL, Sanctuaires et divinités en Helvétie occidentale. Eléments de réflexion sur la typologie des lieux de culte gallo-romains, in: D. Castella / M.-F. MeYlan KRAuSE (Hg.), Topographie sacrée et rituels. Le cas d'Aventicum, capitale des Helvètes, Basel 2008, 229-245.

PAUNIER 1987a = D. PAUNIER u. a., Lousonna 6: Le vicus gallo-romain de LousonnaVidy. Rapport préliminaire sur la campagne de fouilles 1984 (Cahiers d'archéologie romande 40), Lausanne 1987.

PAUNIER $1987 b=$ D. PAUNIER u. a., Du nouveau à l'ouest de Lousonna - bilan de trois années de recherches, AS 10, 1987, 112-125.

PAUNIER 1989 = D. PAUNIER u.a., Le vicus gallo-romain de Lousonna-Vidy. Le quartier occidental, le sanctuaire indigène. Rapport préliminaire sur la campagne de fouilles 1985, Lausanne 1989.

STIFTER 2010/11 = D. STIFTER, Der Schwund von auslautenden s als westeuropäische areale Erscheinung, Die Sprache 49.2, 2010/11, 187-193.

STIFTER 2012 = D. STIFTER, Leniation of s in Gaulish?, in: B. Nielsen Whitehead u.a. (Hg.), The Sound of Indo-European. Phonetics, Phonemics, and Morphophonemics, Copenhagen 2012, 523-544.

\section{Kontakt zum Autor:}

Dr. Jens Bartels

Historisches Seminar der Universität Zürich

- Alte Geschichte -

Karl Schmid-Str. 4

CH - 8006 Zürich

E-Mail: jens.bartels@hist.uzh.ch

\section{(c) $)$ EY}

Dieser Beitrag ist lizenziert unter einer Creative Commons Namensnennung - 4.0 International Lizenz. 